\title{
A Micropipet Aspiration System to Measure the Kinetics of Selectin/Ligand Interactions
}

\section{Jun Huang, Juan Chen, Mian Long}

National Microgravity Laboratory/CAS, Institute of Mechanics, Chinese Academy of Sciences, Beijing 100080, China, Email: mlong@imech.ac.cn

\section{Cheng Zhu}

George W. Woodruff School of Mechanical Engineering and Wallace H. Coulter Department of Biomedical Engineering, Georgia Institute of Technology, Atlanta, GA 30332, USA, Email: cheng.zhu@me.gatech.edu

\begin{abstract}
A micropipet aspiration assay was set up to measure the kinetics of selectin binding to carbohydrate ligands. Reaction rates and binding affinity were estimated from the experimental data using a small system probabilistic model. This work can be extended to analyze kinetics of receptor-ligand binding in many physiological and pathological processes, such as rolling of neutrophils on endothelium.
\end{abstract}

Keywords: Force, probabilistic model, kinetic rates, binding affinity

\section{Introduction}

Receptor/ligand interactions are important to many physiological and pathological processes such as inflammatory reaction, tumor metastasis, etc. For example, selectin/carbohydrate ligand binding have been found to mediate the rolling of leukocytes on endothelial monolayer, the first step of inflammatory cascade, due to the fast kinetic rates [4]. Kinetic rate and binding affinity constants are essential determinants of cell adhesion. Not until recently has kinetic measurements become experimentally possible for receptor/ligand binding on apposed surfaces, so-called two-dimensional (2D) interaction. Using a well-developed small system probabilistic model and a micropipet aspiration assay $[1-3,5]$, we previously measured the Eselectin/ligand binding, and compared the 2D kinetics of E-selectin binding to carbohydrate ligands expressed on a human promyelocytic leukemia cell line (HL-60) with ones expressed on a human colon adenocarcinoma cell line (Colo-205) [3]. Here we further measured the kinetics of P-selectin/ligand interaction, and compared to that of E-selectin/ligand binding.

\section{Materials and Methods}

Micropipet aspiration assay has been described previously. Briefly, it employs an aspirated human red blood cell (RBC) as an ultra-sensitive force transducer to detect and quantify adhesion mediated by even a single receptor/ ligand bond (Figs. 1A-D). Three different types of data were measured simultaneously, one in each of the three distinct phases of an adhesion test cycle: the adhesion score in the no-load contact phase $\left(t_{0}, t_{1}\right)$, the detachment force in the constant-rate retraction phase $\left(t_{1}, t_{2}\right)$, and the adheison lifetime in the constant-load holding phase $\left(t_{2}, t_{3}\right)$ (Fig. 1E). 
The first phase examines how likely adhesion occurs. At time $t_{0}$ the selectin-coated RBC and the ligand-expressing target cell are brought together and kept in contact for a given period of time. At the end of the contact duration $t_{t}$, the computer-driven piezoelectric translator retracts the pinette until it returns to the starting position at time $t_{2}$. An adhesion, if present at the end of the contact, will result in an elongation of the $\mathrm{RBC}$ upon its retraction, enabling visual detection of the occurrence of adhesion. A binary score is obtained, one if adhesion occurs and zero if not. The second phase concerns the positive adhesion event only. For this, retracting the pipette at a constant rate imposes a linearly increasing force on the selectin/ligand bond(s). If the adhesion breaks up during retraction, the force $f$ at which detachment occurs will be measured from the RBC deflection. The third phase concerns the adhesion that survives the retraction only. If the two cells remain adherent after the pipette returns to its starting position at $t_{2}$, the RBC will be held still to keep the force constant (as set by the RBC deflection). The adhesion lifetime $\tau\left(=t_{3}-t_{2}\right)$ at that force will be recorded by a video timer until the two cells dissociates at $t_{3}$. In this work, anti-P-selectin monoclonal antibodies (mAbs) 1478 and $\mathrm{S} 12$ were coated on the surfaces of human RBCs using $\mathrm{CrCl}_{3}$ protocol, which are used to capture P-selectin constructs with Lec/EFG (P-Lec/EGF) and with whole extracellular domains (sPs), respectively (Fig. IF). Capture mAbs-coated $R B C$ s were then incubated with respective $P$ selectin constructs before binding to cultured HL-60 cells. Binding probability, $P_{3}$, on contact duration. $t$, of selectin/ligand interaction at the contact area, $A_{\mathrm{c}}$, was measured experimentally using the micropipet technique, and the kinetic rates and binding affinity were predicted using the small system probabilistic model [1-3],

$$
P_{\mathrm{a}}=1-\exp \left\{-A_{\mathrm{c}} m_{\mathrm{r}} m_{\mathrm{i}} K_{\mathrm{a}}^{0}\left[1-\exp \left(-k_{\mathrm{r}}^{0} t\right)\right]\right\},
$$

where $K_{\mathrm{a}}^{0}$ and $k_{\mathrm{f}}^{0}$ are zero-force binding affinity and reverse rate, respectively, and $m_{\mathrm{r}}$ and $m_{1}$ the site densities of selectin and ligands respectively.

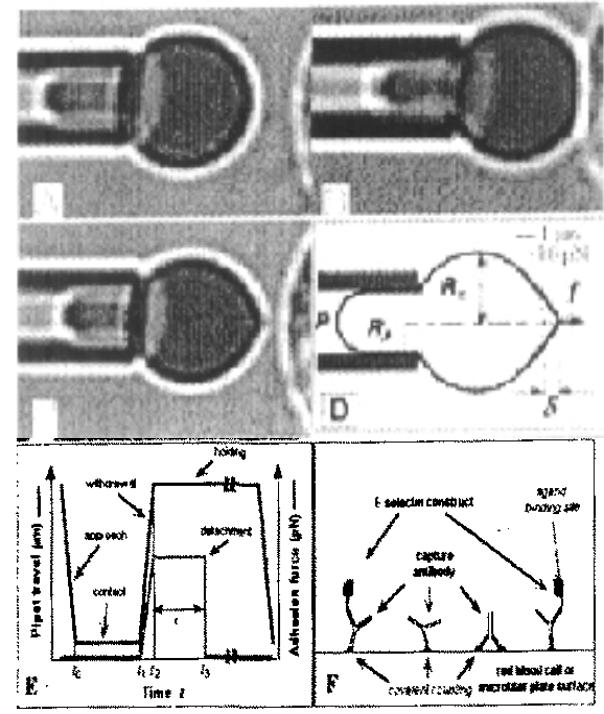

Fig. $1 A-C$ : A micropipette-aspirated RBC coated with $\mathrm{E}$-selectin (left) was driven by a piezoelectric translator to make a controlled contact with the carbohydrate ligand-expressing human colon carcinoma cell (Colo-205), held stationary by another pipette (right, only partially shown). Upon the pipette withdrawal, adhesion was detected from the RBC membrane deflection. $D$ : Overlay drawing of the photomicrographs shown in $A$ and $C$ to illustrate how the adhesion force $f$ can be calculated. $E$ : Schematic of the adhesion test cycle showing the pipette travel (left ordinate) and adhesion force (right ordinate) as functions of time (abscissa). $F$ : Schematic of the chemistry used to coat the E-selectin construct onto the surface of RBC or of the 96-well microtiter plate.

\section{Results}

Control and blocking experiments were designed to address the binding specificity for P-Lec/EGF and SPs, respectively, binding to the ligands on HL-60 cells. Adhesions were abolished when the P-selectin capture mAb 1478 or $\mathrm{S} 12$ was replaced by an isotypematched irrelevant mlgGl or when the Pselectin construct was substituted by the $E$ selectin construct. Adhesions were completely blocked by the anti-P-selectin and anti-PSGL-1 blocking mAbs (PLI). Inclusion of EDTA (5 $\mathrm{mM}$ ) in the medium also abrogated adhesions (data not shown). These experiments showed 
that the binding was specifically mediated by $\mathrm{P}$ selectin/ligand interaction.

A typical curve for dependence of binding probability on contact duration was shown in Fig. 2. Using Eq. 1, the zero-force binding affinity and reverse rate of $\mathrm{P}$-selectin/ligand binding can be estimated from the measured data. These numbers were used to compare with E- and P-selectin dissociating from HL-60 cells, as well as with the data from a flow chamber assay.

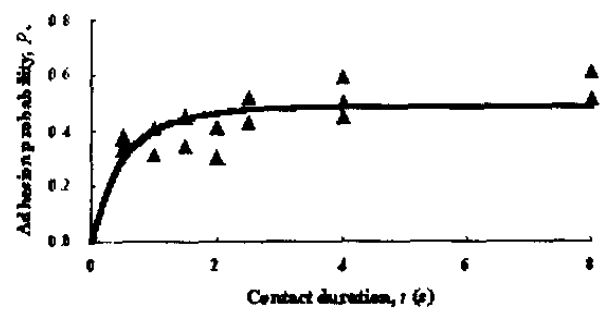

Figure 2. Comparison between measured (points) and predicted (curve) adhesion probability, $P_{\mathrm{a}}$, on contact duration, $t$, for sPs binding to ligands on HL-60 cells. The theoretical prediction is based on Eq. I. Each cell pair was used to repeat 100 cycles to estimate the $P_{\text {a }}$ for that cell pair $(N=17)$.

\section{Acknowledgments}

We thank Rodger P. McEver for generous gifts of the P-selectin constructs and the relative mAbs. Supported by NSFC grants 10042001 and 10072071, a CAS grant KJCX2-L02 and a TRAPOYT Award (ML) as well as by NIH grant AI44902 (CZ).

\section{References}

[1] Chesla, S.E, et al. Measuring two-dimensional receptor-ligand binding kinetics with micropipette. Biophys. J. 75(1998): 1553-1572

[2] Long, M., et al. Probabilistic modeling of shearinduced formation and breakage of doublets cross-linked by receptor-ligand bonds. Biophys. $J$. 76(1998): 1112-1128

[3] Long, M., et al. Kinetic measurements of cell surface E-selectin/carbohydrate ligand interactions. Ann. Biomed. Engi. 29(2001): 935946

[4] McEver, R.P., et al. Leukocyte trafficking mediated by selectin-carbohydrate interactions. $J$. Biol. Chem. 270(1995): 11025-11028.

[5] Zhu, C., et al. Measuring receptor/ligand interaction at the single bond level: Experimental and interpretive issues. Ann. Biomed. Engi. 30(2002): $1-10$ 